Communication

\title{
Magnetocaloric effect of thin Terbium films
}

\author{
V.D. Mello ${ }^{\mathrm{a}, *}$, D.H.A.L. Anselmo ${ }^{\mathrm{b}}$, M.S. Vasconcelos ${ }^{\mathrm{c}}$, N.S. Almeida ${ }^{\mathrm{a}}$ \\ a Departamento de Física Universidade do Estado do Rio Grande do Norte Mossoró-RN RN 59610-210, Brazil \\ b Departamento de Física Teórica e Experimental Universidade Federal do Rio Grande do Norte, Natal, RN 59072-970, Brazil \\ ${ }^{c}$ Escola de Ciência e Tecnologia, Universidade Federal do Rio Grande do Norte, 59072-970 Natal, RN, Brazil
}

\section{A R T I C L E I N F O}

Communicated by S. Das Sarma

Keywords:

Magnetocaloric effect

Rare Earth

Magnetic measurements

Thin Films

\begin{abstract}
A B S T R A C T
We report a theoretical study of the magnetocaloric effect of Terbium ( $\mathrm{Tb})$ thin films due to finite size and surface effects in the helimagnetic phase, corresponding to a temperature range from $T_{C}=219 \mathrm{~K}$ to $T_{N}=231 \mathrm{~K}$, for external fields of the order of kOe. For a Tb thin film of 6 monolayers submitted to an applied field $(\Delta \mathrm{H}=30 \mathrm{kOe}$, $\Delta \mathrm{H}=50 \mathrm{kOe}$ and $\Delta \mathrm{H}=70 \mathrm{kOe}$ ) we report a significative change in adiabatic temperature, $\Delta \mathrm{T} / \Delta \mathrm{H}$, near the Néel temperature, of the order ten times higher than that observed for Tb bulk. On the other hand, for small values of the magnetic field, large thickness effects are found. For external field strength around few kOe, we have found that the thermal caloric efficiency increases remarkably for ultrathin films. For an ultrathin film with 6 monolayers, we have found $\Delta \mathrm{T} / \Delta \mathrm{H}=43 \mathrm{~K} / \mathrm{T}$ while for thicker films, with 20 monolayers, $\Delta \mathrm{T} / \Delta \mathrm{H}=22 \mathrm{~K} / \mathrm{T}$. Our results suggest that thin films of $\mathrm{Tb}$ are a promising material for magnetocaloric effect devices for applications at intermediate temperatures.
\end{abstract}

\section{Introduction}

The magnetocaloric effect (MCE) is a phenomenon that enables the temperature of a material to be altered by the application of external fields. Specifically, a magnetic field acting on a substance alters its magnetic state and, consequently, it changes the internal magnetic energy, so that heat is lost or absorbed reversibly. Under adiabatic conditions, when a magnetic field is switched on or off rapidly, the release or absorption of heat is manifested as an increase or reduction in temperature. Recently, the MCE has attracted the interest of both physicists and engineers: physicists because of potential applications in the study of interactions and changes of magnetic structures in magnetic materials, whereas engineers are hoping to be able to construct new devices and cooling systems [1]. Also, due to the discovery of the giant MCE near room temperature in bulk $\mathrm{Gd}_{5}\left(\mathrm{Si}_{x} \mathrm{Ge}_{1-x}\right)$, by Pecharsky and Gschneidner's [2,3], the interest in the magnetocaloric effect was sparked. This made the prospect of commercializing magnetic cooling to be tenable. Numerous magnetocaloric materials have been studied to date in bulk form [4-7], silicides, transition metal intermetallics, lanthanides, Heusler alloys, and manganites. In contrast, investigating the magnetocaloric effect and related materials remains a novel endeavor for nanoscience or in the limit of ultrathin films. Indeed, exploring the magnetocaloric effect via nanostructuring or ultra thin films, falls securely within the grand challenges of nanomagnetism and their frontiers.
In this context, it is the aim of this work to contribute in some way to the studies of MCE in ultrathin films of rare earth materials. Here we restrict our study to Terbium, due to its large magnetic momentum and interesting magnetic phase transitions (heat capacity of a magnetic material usually shows a peak at its transition temperature) [8]. In a general way, heavy rare earth elements and their compounds are considered to be the best-suited materials for achieving a large MCE [9]. Rare-earth metals have many different magnetic structures resulting from the competition between the crystal field and exchange interactions. When a magnetic field is applied it gives rise to a third interaction and the magnetic structures are more complicated [10-12]. In the absence of an applied magnetic field, for example, the Terbium orders in a basal plane helical phase in the temperature interval from $231 \mathrm{~K}$ (Néel temperature) to $219 \mathrm{~K}$ (Curie temperature). The helix turn angle $\delta \phi$ [13] varies between $20.5^{\circ}$ and $17^{\circ}$. Therefore the helix period corresponds to nearly eighteen atomic layers and one might expect strong surface effects for films in this thickness range. When a magnetic field is applied in the basal plane other magnetic structures are observed including a basal plane ferromagnetic phase, a fan phase and a helifan phase [14]. In thin films, when the thickness is comparable to the periodicity of the ordered structure, it is expected that even the magnetic arrangement itself can be strongly modified. Rare earth helimagnets such as Ho, Dy, and Tb represent the best candidates to put into evidence such finite-size effects. This finite-size effect is caused by the reduced number of atoms in the direction

\footnotetext{
* Corresponding author.

E-mail address: vambertodias@uern.br (V.D. Mello).
} 
perpendicular to the film surface that leads to a decrease of the total magnetic exchange energy.

The MCE is an effect where a variation in the temperature of the magnetic material $(\Delta T)$ is linked to an adiabatic change in an external magnetic field strength. This variation of temperature is a function of both the initial temperature, and the change in the intensity of the applied magnetic field, $\Delta \mathrm{H}=\mathrm{H}_{f}-\mathrm{H}_{i}, \mathrm{H}_{i}$ and $\mathrm{H}_{f}$ are the initial and final measures of the applied field. A positive MCE corresponds to having both $\Delta \mathrm{T}>0$ and $\Delta \mathrm{H}>0$. An alternative definition of the MCE is the change in the magnetic entropy for an isothermal change of the applied field strength.

The reduction of the Zeeman energy splitting of the energy levels, for ferromagnetic materials, results from both effects of reducing the external field, and the reduction of the thermal value of the magnetic moment, when measured in the field direction. Due to the exchange coupling, the internal fields are very large at low enough temperatures, and an applied field affects the magnetic order mainly in the thermal region around the Curie temperature $T_{C}$. In the ordered phase, $d \Delta T / d T>0$, with a maximum value at $T=T_{C}$ [15]. The helimagnetic materials have an abundant phase diagram, and as a consequence a complex MCE arises. In the temperature interval where a magnetic ordering appears, the applied field can modify the magnetic structure, without discernible changes in the average value of the magnetic moment per atom. Also, it is possible to observe both positive and negative MCE, since the effect of an applied field on the magnetic entropy is dependent upon the field strength.

The adiabatic temperature change can be measured directly or indirectly by using heat capacity and magnetization data [16]. In a material displaying MCE, the alignment of randomly oriented magnetic moments by an external magnetic field results in heating of the material. If the magnetic field is subsequently turned off, the magnetic moments randomize again, which leads to cooling of the material. The MCE of the $\mathrm{Gd}_{5}(\mathrm{Si}, \mathrm{Ge})_{4}[17,18]$ have been extensively studied due to their potential use for magnetic refrigeration applications near room temperature, as we have pointed above. These compounds undergo a first-order phase transition and exhibit a large MCE. Measurements of the MCE in polycrystalline Ho [19] displayed MCE maxima at $\mathrm{T}_{C}=20 \mathrm{~K}$ $(\Delta \mathrm{T}=4.6 \mathrm{~K}$ for $\Delta \mathrm{H}=60.2 \mathrm{kOe})$ and at $\mathrm{T}_{N}=132 \mathrm{~K}(\Delta \mathrm{T}=4.5 \mathrm{~K}$ for $\Delta \mathrm{H}=60.2 \mathrm{kOe}$ ). Recent works demonstrated that thin Dy [20] and Ho [21] films display MCE of the same order of magnitude found in giant magnetocaloric effect (GMCE) materials. The enhancement of the MCE, relative to the bulk Dy and Ho values, is more pronounced for small values of the external field strength, where in ultra-thin films, the helical state forms and the film behaves as a ferromagnetic.

In this paper we are interested to investigate the thickness influence on MCE of Terbium thin films, in the frontier between nanometric 620 monolayers) and bulk structures, in the temperature range from $220 \mathrm{~K}$ to $230 \mathrm{~K}$. These films have an hcp crystal structure, characterized by the stacking of atoms in planes of hexagonal symmetry, along the $c$ direction. The space group of $\mathrm{Tb}$ is $\mathrm{P}_{3} / \mathrm{mmc}$. The plan of this paper is: In Section 2 we present the theoretical model used here. Section 3 is devoted to present the results and a discussion about the MCE in Terbium thin films. Finally, in Section 4 we present the conclusions of this paper.

\section{Theoretical Model}

We investigate a c-axis thin film, consisting of a stacking of atomic layers with equivalent spins, infinitely extended in the $x$ - $y$ directions. The spins in each monolayer are exchange-coupled with the spins in the first and second neighbour monolayers, as shown on Fig. 1. The anisotropy is uniform throughout the film and the near-surface spins have reduced exchange energy. The magnetic Hamiltonian is given by:

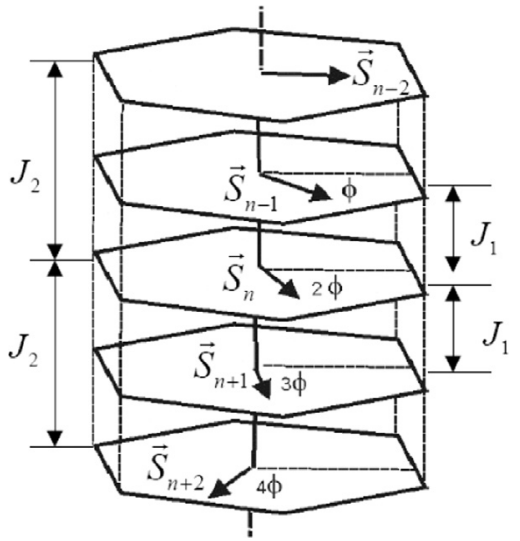

Fig. 1. Magnetic structure of the helix phase of terbium. Our model considers interactions up to next-nearest neighbours.

$$
\begin{aligned}
\mathcal{H}= & J_{1}(g-1)^{2} \sum_{n=1}^{N-1} \vec{J}(n) \cdot \vec{J}(n+1) \\
& +J_{2}(g-1)^{2} \sum_{n=1}^{N-2} \vec{J}(n) \cdot \vec{J}(n+2) \\
& +\sum_{n=1}^{N}\left[K_{6}^{6}(T) \cos \left(6 \phi_{n}\right)-g \mu_{B} \vec{J}(n) \cdot \vec{H}\right]
\end{aligned}
$$

In Eq. (1), $J_{1}$ and $J_{2}$ describe the exchange interaction between the nearest and next-nearest monolayers respectively, $\vec{J}(n)$ denotes the total angular momentum per atom in the $n$-th monolayer. The coefficient $K_{6}^{6}(\mathrm{~T})$ describes the hexagonal anisotropy and the last term is the Zeeman Energy, where the external field $\vec{H}$ is applied in one easy direction in the hexagonal plane, making an angle of $30^{\circ}$ with $x$ axis.

We use the Tb bulk energy parameters [22], where the modulus of the total angular momentum is $J=6, \mathrm{~J}_{1}=47 k_{B}, \mathrm{~J}_{2}=-J_{1} / 4 \cos \phi(T)$, where $\phi(T)$ is the temperature dependent helix turn angle [23], $\mathrm{g}=3 / 2$ is the Landé factor, corresponding to a saturation magnetic moment per atom of $9.7 \mu_{B}$. The parameter $K_{6}^{6}$ is obtained from the fit of the experimental curves of the temperature dependence of the hexagonal anisotropy [24].

We use a self-consistent local field model which incorporates the surface modifications in the exchange field and the thermal average values $(\langle J(n)\rangle ; n=1 \ldots N)$ and the orientation of the spins in each layer $\left(\left\langle\phi_{n}\right\rangle ; n=1 \ldots N\right)[25,26]$. However, the surface effects are not necessarily restricted to the surface layers. The number of layers modified by surface effects depends on the way the effective local field relaxes towards the bulk pattern in the middle of the film.

We calculate the MCE numerically as a function of the magnetization. If the magnetization is a continuous function of the temperature and magnetic field, then the adiabatic temperature change is given by:

$\Delta T_{a d}=-\int_{H_{i}}^{H_{f}} \frac{T}{C(T, H)_{H}}\left(\frac{\partial M(T, H)}{\partial T}\right)_{H} d H$

From Eq. (2) it is easy to state that a material should have large MCE when $\left(\frac{\partial M(T, H)}{\partial T}\right)_{H}$ is large and $C(T, H)$ is small, at the same temperature [27]. Starting the process from $H_{i}=0$ to $H=H_{f}$ we obtain $\Delta T_{a d}(T, H)$. Here $\left(\frac{\partial M(T, H)}{\partial T}\right)_{H}$ is the temperature derivative of the magnetization along the external field direction and $C(T, H)$ is the heat capacity expressed by [28]:

$C(T, H)=T\left(\frac{\partial S(T, H)}{\partial T}\right)_{H}$

where the total entropy $S(T, H)$ of a magnetic material is, at constant pressure, the sum of the lattice, electronic and magnetic contributions:

$S(T, H)=S_{\text {Mag }}(T, H)+S_{\text {Latt }}(T, H)+S_{\text {Elet }}(T, H)$ 
In this work we ignore the field dependence of the electronic entropy (due to its small contribution to the total entropy). Thus the magnetic contribution is given by:

$S_{\text {Mag }}=\frac{1}{T} \sum_{s=1}^{2 J+1} \frac{\mathcal{H} e^{-\mathcal{H} / k_{B} T}}{Z}+k_{B} T \ln Z$

where $Z=\sum e^{-\mathcal{H} / k_{B} T}$ is the partition function, $\mathcal{H}$ is the Hamiltoninan that describes the energy of the system (given by Eq. (1)), $T$ is the temperature and $k_{B}$ is the Boltzmann constant. The lattice contribution is given by:

$S_{\text {Latt }}=-3 R \ln \left[1-e^{\Theta_{D} / T}\right]+12 R\left(\frac{T}{\Theta_{D}}\right)^{3} \int_{0}^{\Theta_{D} / T} \frac{x^{3} d x}{e^{x}-1}$

where $R$ is the gas constant and $\Theta_{D}$ is the Debye temperature, which for $\mathrm{Tb}$ is $\Theta_{D}=158 \mathrm{~K}$ [22]. For a review on the magnetocaloric effect with special attention to nanoscale thin films and heterostructures see ref. $[29,30]$.

\section{Results and Discussion}

In this section we show the numerical results of the MCE, for different applied fields and thicknesses of Tb thin films. Our studies revealed that the efficiency of the MCE in nanofilms of Tb has a strong dependence with its thickness. We note, too, that dependence of field in MCE decreases continuously with increasing thickness of film, approaching the standard value for bulk when the film becomes too thick (in a nanometric scale).

We show on Fig. 2 the variation of $\Delta T_{a d}(K)$ for films of Tb with 6,10 and 20 monolayers for an external field of $\Delta H=1 \mathrm{kOe}$. We have chosen a small value of $\mathrm{H}$ because the thin film effects on the MCE properties are more visible for small values of the external field. We found significant $\Delta T(T, H) / \Delta H$ values: $43 K / T$ for 6 monolayers thin film, $40 \mathrm{~K} / \mathrm{T}$ for 10 monolayers thin film and $22 \mathrm{~K} / \mathrm{T}$ for 20 monolayers thin film.

In order to stress the thickness influence on the MCE, we show on Fig. 3 the variation of $\Delta T_{a d}(K)$ for a 6 monolayer Tb film and for bulk $\mathrm{Tb}$, at an external field of $\Delta H=1 \mathrm{kOe}$. We found that the peak value of $\Delta T_{a d}(K)$ for the thin film is nearly two times larger than the corresponding bulk value.

We can also observe, Figs. 2 and 3, the dependence of the Nel temperature with the film thickness, where for thick films it approximates bulk $T_{N}$ and for thinner films the temperature decreases. Near the Nel temperature the bulk and thin film values of the helix turn angle are similar, $\phi(T) \approx 20^{\circ}$. Consequently, a complete helix corresponds to around eighteen atomic layers. One might then expect to find

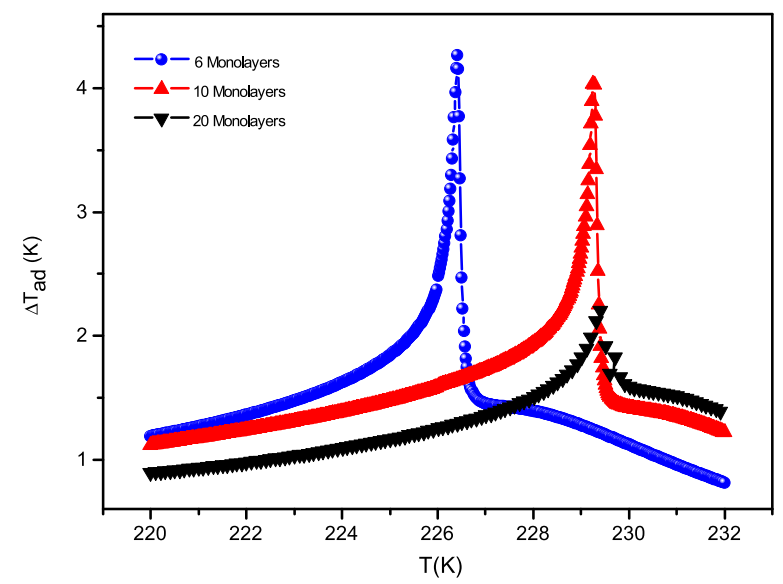

Fig. 2. (Color online) Adiabatic variation of the temperature as a function of $\mathrm{T}$ for a Terbium film with thickness of 6,10 and 20 monolayers, for an applied field of $\Delta \mathrm{H}=1$ kOe.

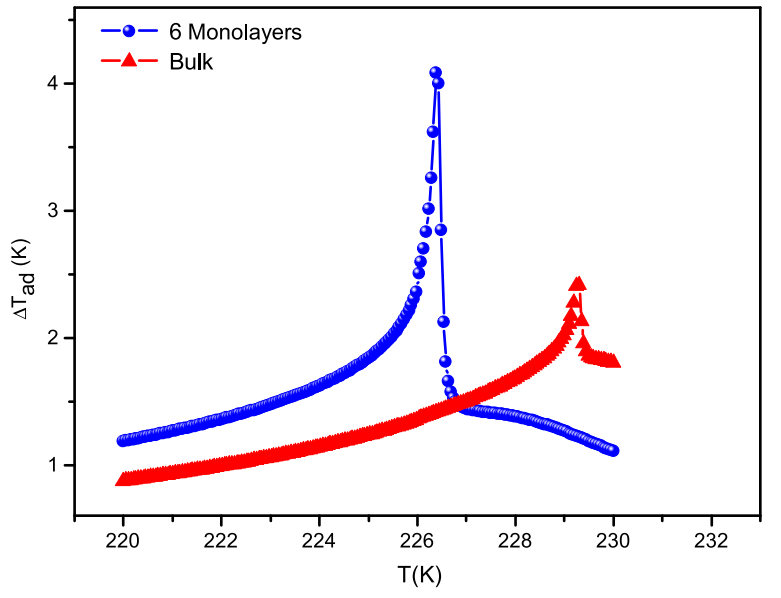

Fig. 3. (Color online) Adiabatic variation of the temperature as a function of $\mathrm{T}$ for a Terbium film with thickness of 6 monolayers and bulk, for an applied field of $\Delta \mathrm{H}=1 \mathrm{kOe}$.

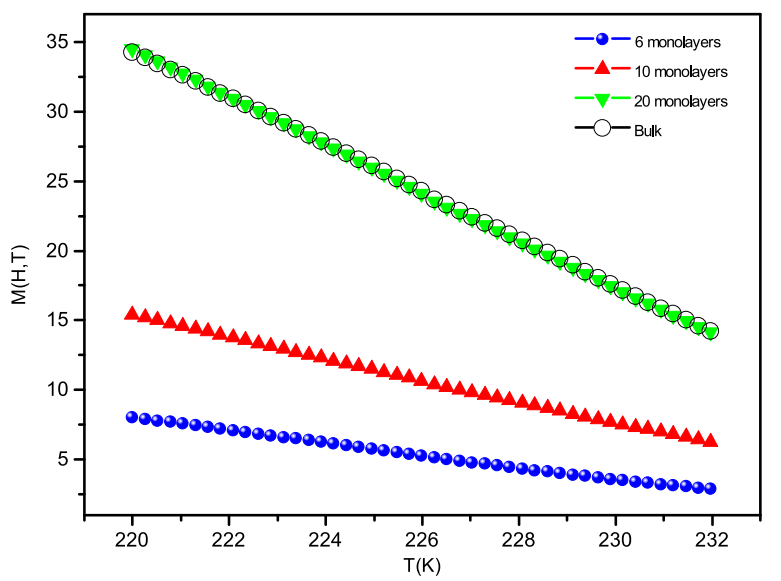

Fig. 4. (Color online) Isofield magnetization curves for external field $H=1$ kOe. The magnetization is shown in units of $\mu_{B}$.

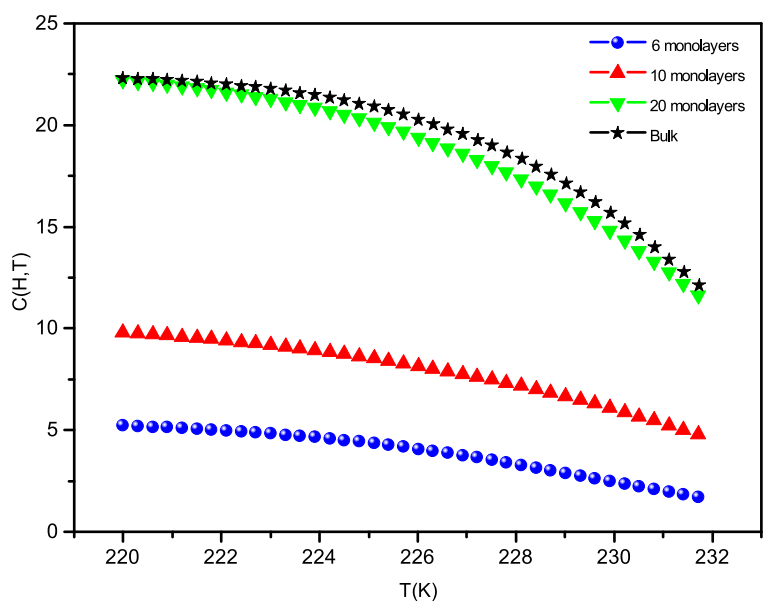

Fig. 5. (Color online) $\mathrm{C}(\mathrm{H}, \mathrm{T})$ for an applied field of $\Delta \mathrm{H}=1 \mathrm{kOe}$.

bulk MCE properties for films with more than 20 monolayers.

The key feature for thin film effects is the field dependence of the field derivative of the adiabatic temperature rise, as seen from Eq. (2), $(d T / d H)_{H}=-T(\partial M(T, H) / \partial T)_{H} / \mathrm{C}(\mathrm{T}, \mathrm{H})_{H}$. The number of modified layers by the surface effect depends on the way the local effective field relaxes the spins in the middle of film. In this manner, changes will occur in 


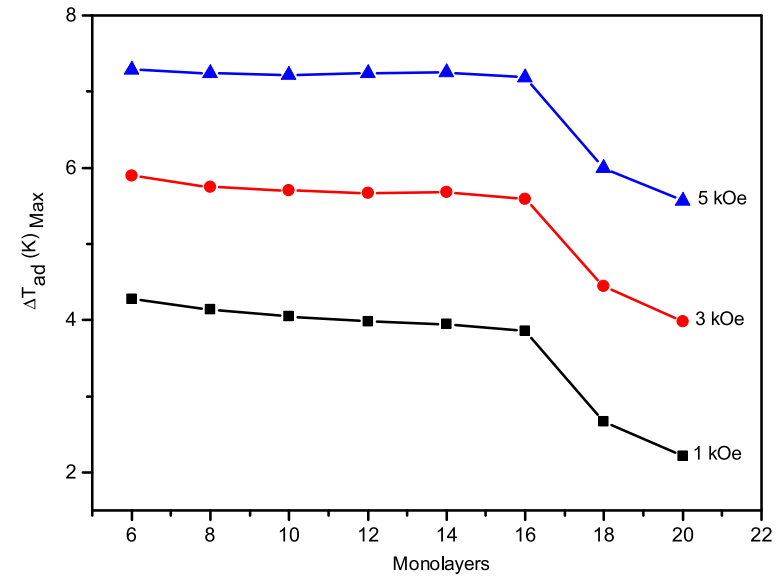

Fig. 6. (Color online) Monolayers dependence of the value $\Delta T(T, H)_{\text {Max }}$ for an external field $\Delta H=1 \mathrm{kOe}, \Delta H=3 \mathrm{kOe}$ and $\Delta H=5 \mathrm{kOe}$.

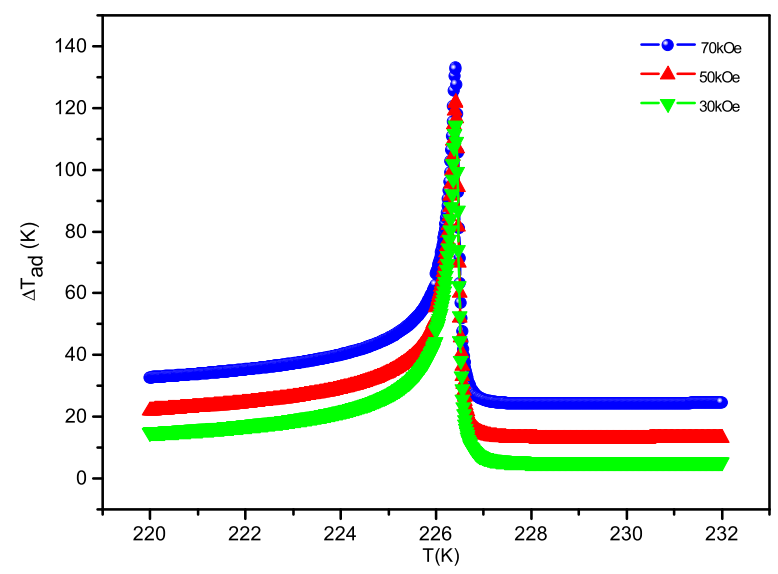

Fig. 7. (Color online) Adiabatic variation of the temperature as a function of $\mathrm{T}$ for a Terbium film with 6 monolayers for applied fields $(\Delta \mathrm{H}=30 \mathrm{kOe}, \Delta \mathrm{H}=50 \mathrm{kOe}$ and $\Delta \mathrm{H}=70 \mathrm{kOe})$.

magnetic phases of thin films, compared with the bulk and consequently on the values of $(\partial M(T, H) / \partial T)_{H}$, significantly affecting the value of $\Delta T_{a d}$. As a result, the adiabatic temperature change is larger than the corresponding value for bulk.

On Figs. 4 and 5 we show the contribution of isofield magnetization and heat capacity for $\Delta T_{a d}(K)$ for films of Tb with $6,10,20$ monolayers and bulk, for an external field of $\Delta H=1 \mathrm{kOe}$, where we can observe that for thicker films, these contributions resemble those of bulk. This fact makes the $\Delta T_{a d}(K)$ of thick films close to that of volume, imposing a theoretical and practical approximation for MCE simulations in $\mathrm{Tb}$ nanofilms.

We show in Fig. 6 that the MCE efficiency, $\Delta T(T, H) / \Delta H$, increases as the thickness is reduced. For instance, for a thin film of 6 monolayers, we have approximately: $43 K / T, 19 K / T$ and $14 K / T$ for external applied fields of $\Delta H=1 \mathrm{kOe}, \Delta H=3 \mathrm{kOe}$ and $\Delta H=5 \mathrm{kOe}$, respectively. For a thin film of 18 monolayers, we will have efficiencies of approximately: $26 \mathrm{~K} / \mathrm{T}, 15 \mathrm{~K} / \mathrm{T}$ and $12 \mathrm{~K} / \mathrm{T}$ for external applied fields of $\Delta H=1 \mathrm{kOe}, \Delta H=3 \mathrm{kOe}$ and $\Delta H=5 \mathrm{kOe}$, respectively. Therefore, the finite size enhancement of the MCE is larger for a small field strength.

On Fig. 7 we depict the adiabatic temperature variation for a $\mathrm{Tb}$ film of 6 monolayers subjected to external fields $(30 \mathrm{kOe}, 50 \mathrm{kOe}$ and $70 \mathrm{kOe})$, with values of $\Delta T(T, H) / \Delta H$ equal to $39 K / T, 25 K / T$ and $19 K / T$, respectively, where we observed that the dependence of the MCE on the applied field is much stronger than the obtained for bulk
Tb. Near the Nel temperature, the value found for $\Delta T_{a d}$ is approximately ten times higher than that obtained for polycrystalline samples of $\mathrm{Tb}$ [22]. This effect is referred as giant magnetocaloric effect (GMCE), a name proposed by Pecharsky and Gschneidner in 1997 as they investigate $\mathrm{Gd}_{5} \mathrm{Si}_{2} \mathrm{Ge}_{2}$ [16]. From the previous results we can conclude that $\mathrm{Tb}$ thin films are indeed a promising magnetocaloric material for applications at intermediate temperatures. From the experimental point of view, the standard procedure to grow the $\mathrm{Tb}$ films is the molecular beam epitaxy (MBE) method [31,32]. The film is grown on a sapphire substrate where it is deposited niobium $(\mathrm{Nb})$ to provide a chemical buffer between the sapphire and the reactive rare earths. Finally, a layer of nonmagnetic yttrium (Y) is deposited on the $\mathrm{Nb}$ to provide a structure with a growth plane with similar structure and a grid parameter for the basal plane of the rare earth layers.

\section{Conclusions}

In conclusion, the present study demonstrated that the MCE efficiency of $\mathrm{Tb}$ is significantly enhanced by finite size and surface effects. Confinement in rather thin films, with thickness below the helix period, favors a ferromagnetic state. This is due to the fact that nearsurface spins are more easily turned in the direction of the external field. Near the surfaces the turn angle is smaller, due to the lack of second neighbors which favors a ferromagnetic-like configuration in the whole temperature range from the Curie temperature to the Nel temperature, leading to a giant MCE. The present results suggests that thin films of $\mathrm{Tb}$ might be a promising material of MCE devices for applications at intermediate temperatures, for example, the cooling of natural gas to liquid form, so that it can be cost-effectively shipped to long distances. Finally, we hope that our results can stimulate the experimentalists to probe the findings presented here.

\section{Acknowledgments}

The authors acknowledge the financial support from the Brazilian research agencies CNPq and FAPERN (No 2139/2013, Processo No 23038.007527/2013-66).

\section{References}

[1] Casey W. Miller, D.V. Williams, N.S. Bingham, H. Srikanth, J. Appl. Phys. 107 (2010) 09A903.

[2] V.K. Pecharsky, K.A. Gschneidner Jr., Phys. Rev. Lett. 78 (1997) 4494.

[3] V.K. Pecharsky, K.A. Gschneidner Jr., Appl. Phys. Lett. 70 (1997) 3299.

[4] M.H. Phan, S.C. Yu, J. Magn. Magn. Mater. 308 (2007) 325.

[5] N.K. Singh, K. Suresh, A. Nigam, S. Malik, A. Coelho, S. Gama, J. Magn. Magn. Mater. 317 (2007) 68.

[6] E. Brück, O. Tegus, D.C. Thanh, N.T. Trung, K. Buschow, Int. J. Refrig. 31 (2008) 763.

[7] Lingwei Li, Yalin Yi, Kunpeng Su, Yang Qi, Dexuan Huo, R. Pöttgen, J. Mater. Sci. 51 (2016) 5421.

[8] H. Drulis, A. Hackemer, L. Folcik, A. Zaleski, Solid State Commun. 150 (2010) 164.

[9] A.M. Tishin, J. Appl. Phys. 68 (1990) 6480.

[10] V.D. Mello, A.S. Carriço, Surf. Sci. 482 (2001) 960.

[11] F.H.S. Sales, A.L. Dantas, V.D. Mello, A.S. Carriço, J. Mater. Sci. 45 (2010) 5036.

[12] L.J. Rodrigues, V.D. Mello, D.H.A.L. Anselmo, M.S. Vasconcelos, J. Magn. Magn. Mater. 377 (2015) 24.

[13] S. Kobayashi, Phys. Rev. B 84 (2011) 224418.

[14] J. Jensen, A.R. Mackintosh, Rare Earth Magnetism, Oxford University Press, Oxford, 1991.

[15] V.K. Pecharsky, K.A. Gschneidner, J. Magn. Magn. Mater. 200 (1999) 44.

[16] V.K. Pecharsky, K.A. Gschneidner, J. Appl. Phys. 86 (1999) 565.

[17] L. Morellon, J. Blasco, P.A. Algarabel, M.R. Ibarra, Phys. Rev. B 62 (2000) 1022.

[18] J.D. Moore, G.K. Perkins, Y. Bugoslavsky, L.F. Cohen, M.K. Chattopadhyay, S.B. Roy, P. Chaddah, K.A. Gschneidner Jr., V.K. Pecharsky, Phys. Rev. B 73 (2006) 144426.

[19] S.A. Nikitin, A.S. Andreenko, A.M. Tishin, A.M. Arkharov, A.A. Zherdev, Phys. Met. Metallogr. 60 (1985) 56.

[20] V.D. Mello, A.L. Dantas, A.S. Carriço, Solid State Commun. 140 (2006) 447.

[21] F.C. Medeiros, V.D. Mello, A.L. Dantas, F.H.S. Sales, A.S. Carriço, J. Appl. Phys. 109 (2011) 07A914. 
[22] A.M. Tishin, Y.I. Spichkin, The Magnetocaloric Effect and its Applications, IOP Publ. Ltd., Bristol, 2003.

[23] O.W. Dietrich, J. Als-Nielsen, Phys. Rev. 162 (1967) 315.

[24] B. Coqblin, The Electronic Structure of Rare-Earth Metals and Alloys: the Magnetic Heavy Rare-Earths, Academic Press, New York, 1977.

[25] R.E. Camley, Phys. Rev. B 35 (1987) 3608.

[26] A.L. Dantas, R.E. Camley, A.S. Carriço, Phys. Rev. B 75 (2007) 094436.

[27] E. Bruck, J. Phys. D: Appl. Phys. 38 (2005) R381.
[28] K.A. Gschneidner Jr, V.K. Pecharsky, A.O. Tsokol, Rep. Prog. Phys. 68 (2005) 1479.

[29] W. Casey, D.D. Belyea, B.J. Kirby, J. Vac. Sci. Tech. A 32 (2014) 040802.

[30] K.A. Gschneidner Jr., V.K. Pecharsky, Annu. Rev. Mater. Sci. 30 (2000) 387.

[31] J.A. Simpson, R.A. Cowley, D.F. McMorrow, R.C.C. Ward, M.R. Wells, C.J. Carlile, M.A. Adams, J. Phys. Condens. Matter 8 (1996) L187.

[32] K.T. Bröhl, K.A. Ritley, C.P. Flynn, J.E. Van Nostrand, D.G. Cahill, K. Hamacher, H. Kaiser, J.J. Rhyne, J. Magn. Magn. Mater. 166 (1997) 27. 